\title{
GenomePlex Whole Genome Amplification
}

National Cancer Institute

\section{Source}

National Cancer Institute. GenomePlex Whole Genome Amplification. NCI Thesaurus.

Code C128787.

A proprietary method for whole genome amplification (WGA) from very small samples using random fragmentation of genomic DNA and conversion of the resulting small fragments to a library of PCR-amplifiable molecules flanked by universal priming sites. 